\title{
FIBROCYSTIC DISEASE OF THE PANCREAS WITH MECONIUM PERITONITIS AT BIRTH
}

\author{
BY \\ F. W. NASH and J. F. SMITH \\ From the Children's Department and the Bernhard Baron Institute of Pathology, The London Hospital
}

(RECEIVED FOR PUBLICATION JUNE 22, 1951)

The relationship between meconium ileus and congenital fibrocystic disease of the pancreas is well recognized. Fibrocystic disease presenting with meconium peritonitis at birth is much less common and it is, therefore, considered justifiable to record a single case, especially as on this occasion the histological examination has been supplemented by serial sections of the main pancreatic ducts.

\section{Case Report}

L.M., a male infant, was delivered spontaneously on August 21, 1949, at full term, after a normal pregnancy, and weighed $6 \mathrm{lb} .10 \mathrm{oz}$. Both parents and one sibling were normal and healthy. At birth the infant's abdomen was large and tense (girth $37 \mathrm{~cm}$. at six hours) and a right hydrocoele was present. He showed a mild degree of asphyxia, but respirations were established satisfactorily within a few minutes of birth. Shortly after delivery the child passed a single stool which was said to be 'mudcoloured', and he regurgitated mucus once. Because of the abdominal distension he was transferred to The London Hospital 30 hours after delivery. On admission his general condition was very good; temperature $98^{\circ} \mathrm{F}$. pulse 140, weight $6 \mathrm{lb} .7 \mathrm{oz}$. There had been no further vomiting or passage of meconium. The abdomen was considerably enlarged and there were dilated veins over the upper abdomen and lower chest wall. There was an obvious fluid thrill in the abdomen, but no guarding or rigidity. Deep palpation was not possible because of the degree of distension, and on auscultation bowel sounds could not be heard. The umbilical stump was clean. The other systems were normal.

A blood count gave Hb. $133^{\circ}{ }_{\mathrm{o}}\left(19.7 \mathrm{~g} .{ }^{\circ}{ }_{\mathrm{o}}\right)$ : leucocytes 20,000 per c.mm (neutrophil-polymorphs $72 \%$, lymphocytes $25^{\circ}$, eosinophils $2^{\circ} .0$, monocytes $1^{\circ}$ ).

A radiograph of the abdomen showed a large aircontaining loculus with a fluid level in the right upper quadrant, extending as high as the dome of the diaphragm (Fig. 1). A diagnosis of intestinal obstruction was made, and it was thought that peritonitis was probably also present.

The child was given a slow intravenous drip of $\frac{1}{5}$ normal saline in $4 \%$ glucose solution, and a laparotomy under general anaesthesia was performed by Mr. J. E.
Richardson. Large quantities of turbid brown fluid escaped as soon as the peritoneal cavity was opened. The loops of the small intestine were matted together and were closely adherent to the left side of the abdomen. There was no evidence of distension of the bowel and no perforation was seen, although adequate exploration of the abdomen was impossible.

The child died within a few hours of the operation.

Microscopy of the peritoneal fluid showed the presence of numerous pus cells, and on culture a pure growth of Bact. coli was obtained.

\section{Necropsy Report}

The findings of the necropsy (P.M.311/49) may be summarized as general peritonitis, perforation of the transverse colon and cystic fibrosis of the pancreas.

Peritoneal Cavity. No free fluid was present but coils of small intestine and part of the large intestine

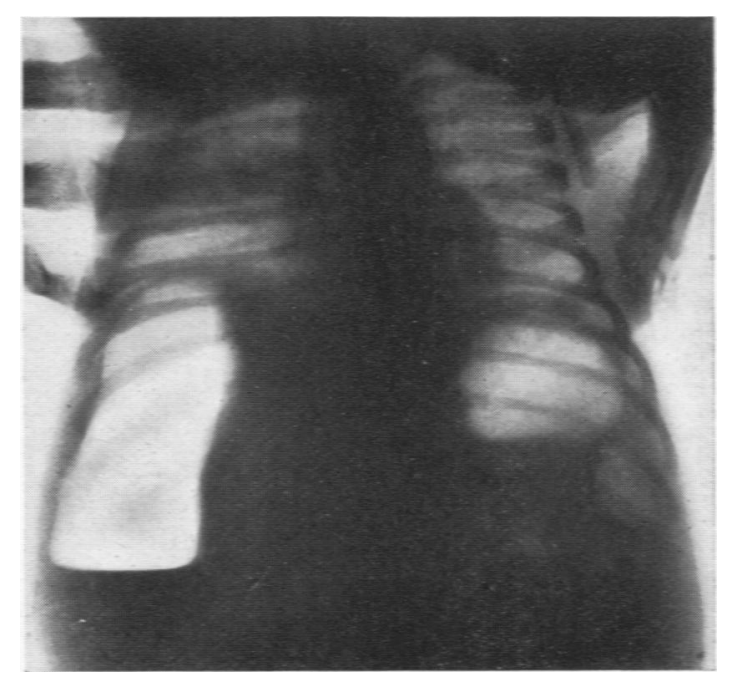

Fig. 1.-Radiograph of abdomen showing air and fluid level beneath right dome of diaphragm. 
were united by stiff, greenish-brown exudate (up to $0.2 \mathrm{~cm}$. thick) suggesting fibrin containing some meconium. A similar exudate was present on the anterior and inferior surfaces of the right lobe of the liver, in patches on the parietal peritoneum of the right side and in the left iliac fossa, but was scanty in the left hypochondrium.

Alimentary Tract. There was a perforation $(0.2 \mathrm{~cm}$. diameter $)$ in the wall of the transverse colon near the splenic flexure, the margin on the serosa being slightly raised and surrounded by a zone $(0.1 \mathrm{~cm}$. wide) of prolapsed mucosa : on the mucosal side the hole was filled by a plug of inspissated meconium. Similar meconium in small pieces was seen in the jejunum, and in larger pieces in the caecum and appendix, but was very scanty elsewhere and absent from the rectum: all of it was yellowishbrown. firm and stiff, the usual slimy consistency being noticeably absent. There was slight distension (up to $2.5 \mathrm{~cm}$. diameter) of the rather turgid lower ileum but not of the remaining intestine. There was no evidence of obstruction within or outside the lumen of any part of the intestine.

There was slight congestion of the pancreas. No obstruction to the common bile duct was seen. There were orange uratic streaks in the medulla of the kidneys. There was central congestion of the suprarenals, with haemorrhage in the capsule of the right. A few petechiae were seen on the surface of the thymus and thyroid. There were scattered patches of collapse in congested lungs, but no abnormality in the trachea and main bronchi. A few millelitres of greenish-yellow fluid were found in both pleural cavities and in the pericardial sac. There was a centre of ossification $(0.5 \mathrm{~cm}$. diameter) at the lower end of the femur. The body was that of a normal-sized, three-day-old male ( $5 \mathrm{lb}$. $13 \frac{1}{2} \mathrm{oz}$.).

Weights of Organs. Heart, 16 g.; liver, 112 g.; kidney, 27 g.; suprarenals, 9 g.; spleen, 6.8 g.; brain, 427 g.; thyroid, 1.4 g.; thymus, 4.4 g.; testis, $0.5 \mathrm{~g}$.

Microscopical Examination. Portions of the pancreas (head, body and tail), lung, right middle and lower lobes, left lower lobe, trachea, stomach with cardia, lower oesophagus, appendix, colon at site of perforation, testis, thyroid, right and left suprarenals, prostate, thymus, kidney, liver, right lobe, spleen and rib were fixed in formol-saline, embedded in paraffin, and stained with haematoxylin and eosin and with iron haematoxylin and van Gieson. A selection of tissues was also stained by the periodic-acid-Schiff (P.A.S.) method using watery solutions. In addition serial sections were made of the head of the pancreas and adjacent duodenum to include the terminal parts of the main pancreatic and common bile duct.
The most important finding was the fibrocystic change in the pancreas. The glands of the trachea and main bronchi contained abundant mucin: changes in other organs were slight.

Pancreas. All sections showed a severe degree of fibrocystic disease, the changes being of uneven degree in different parts of the organ and in different parts of any one section but on the whole most advanced in the head. There was considerable dilatation of interlobular and interacinar ducts, their epithelium being flattened and their lumina empty or containing bright eosinophil secretion (Fig. 2); in a few there were collections of partly necrotic polymorphonuclear leucocytes. In places the individual cells of the acini were atrophied; only a few acini contained retained secretion. There was widespread oedema, and patchy fibrosis between acini was more marked between lobules (Fig. 3) and associated with foci of dilated capillaries and lymphocytic infiltration. Acute inflammation was not seen in the interstitial tissue. The islets of Langerhans were not conspicuous but they showed no change.

In serial sections of the head and attached duodenum the mucosa of the common opening of the duct of Wirsung and the bile duct was deeply folded; at 375-750 $\mu$ from the tip of the ampulla the lumen of the bile duct was star-shaped, that of the duct of Wirsung appearing as numerous spaces between columns of fibrous tissue (Fig. 4). Further back the ducts were round or oval with small diverticula branching from the main lumina, these being more prominent in relation to the bile duct. The main pancreatic duct was not dilated, and at $2.6 \mathrm{~mm}$. from the tip of the ampulla it could not be distinguished with certainty from some of its branches. There was no evidence that the duct of Wirsung was stenosed; the appearance in Fig. 4 is probably due to partial tangential section of a plicated epithelium. (A plicated epithelium was seen in the terminal portion of the main pancreatic duct in material obtained from a control full term foetus, and in places the duct also appeared as multiple spaces in section.)

Alimentary Tract, Spleen and Liver. In the section through the perforation in the transverse colon the mucosa was prolapsed through to the serosa (Fig. 5) with very little reaction about it. The adjacent muscularis was oedematous as in the section of the appendix. One gland in the mucosa of the appendix was considerably dilated with retained secretion. The peritoneum over the appendix, that over the capsule of the liver and one area over the spleen showed an organizing exudate in which histiocytes, lymphocytes, dilated capillaries, a little delicate collagen and a very few small foreign 
body giant cells were present (Fig. 6). Also in the exudate, and presumably derived from meconium. were some pigmented bodies, a few epithelial squames and a very few refractile bodies of uncertain nature, some of which were related to the foreign body giant cells. There was slight fatty change in the parenchyma of the liver and congestion of the splenic pulp.

Respiratory System. The section of the left lower lobe showed areas of collapse. Both here and in the right middle and lower lobes many bronchi and alveolar spaces contained hyaline, eosinophil, amorphous necrotic material including refractile squames and occasional foam cells containing sudanophil droplets. There was abundant mucin in the cells and lumina of glands related to the main bronchus to the left lower lobe; one of these was dilated (Fig. 7). A transverse section of the trachea showed similar abundant mucin and also focal necrosis of its mucosa with replacement of the latter by inflammatory tissue.

Other organs showed no significant changes.

\section{Discussion}

The cause of death in this case was clearly the peritonitis, and this was obviously due to the leakage of meconium through the perforation in the transverse colon. The appearances of the exudate indicated that it was at least three or four days old. which would be compatible with a perforation occurring shortly before or during delivery.

Peritonitis occurring in the child before birth or in the first few days of life is a very uncommon event. Simpson (1838), however, reported details of 25 cases, and in recent years Abt (1931) and Thelander (1939) have published extensive reviews of the subject. The majority of case reports do not indicate clearly the aetiology of the condition, although in most there has been evidence of an escape of meconium into the peritoneal cavity.

Meconium peritonitis can result from leakage through a congenital defect in the intestinal wall, such as a patent Meckel's diverticulum, but is more often a sequel to intestinal obstruction. The latter may be due to mechanical factors such as congenital

FIG. 2.-Retained secretion within a dilated duct of the pancreas, round cell infiltration in surrounding connective tissue. H. \& E. 130.

FIG. 3.-Fibrosis between and within lobules of the pancreas. Van Gieson · 82.

Fig. 4.-Terminal part of duct of Wirsung (numerous small spaces) and of common bile duct (star-shaped) in partial tangential section about $500 \cdot \Omega$ from tip of the ampulla of Vater. Van Gieson . 17.

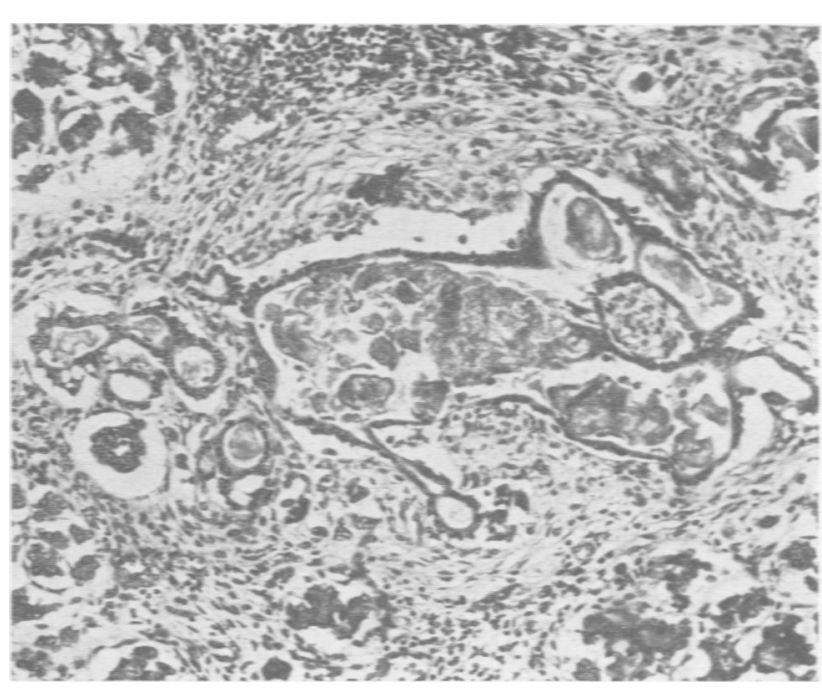

FIG. 2.

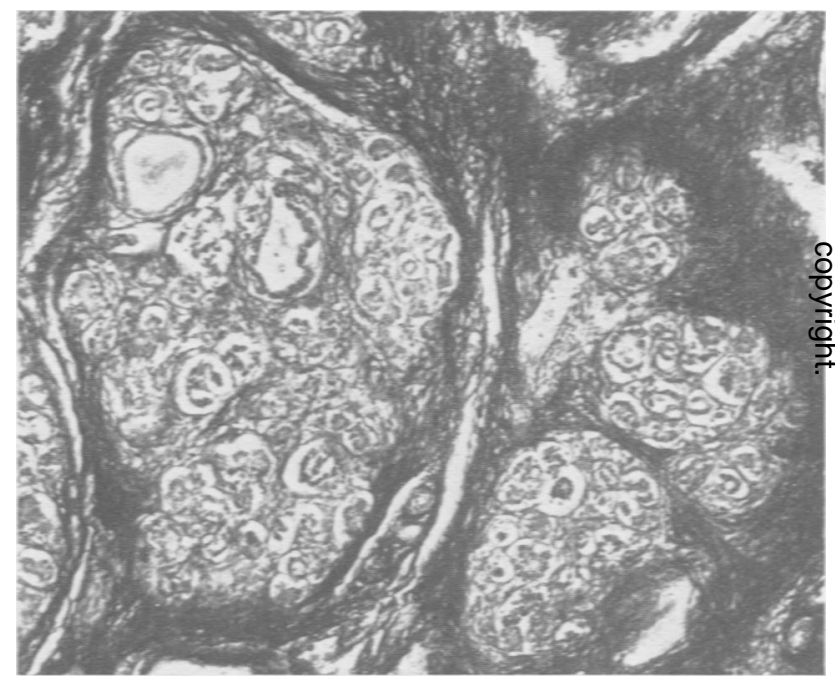

Fig. 3.

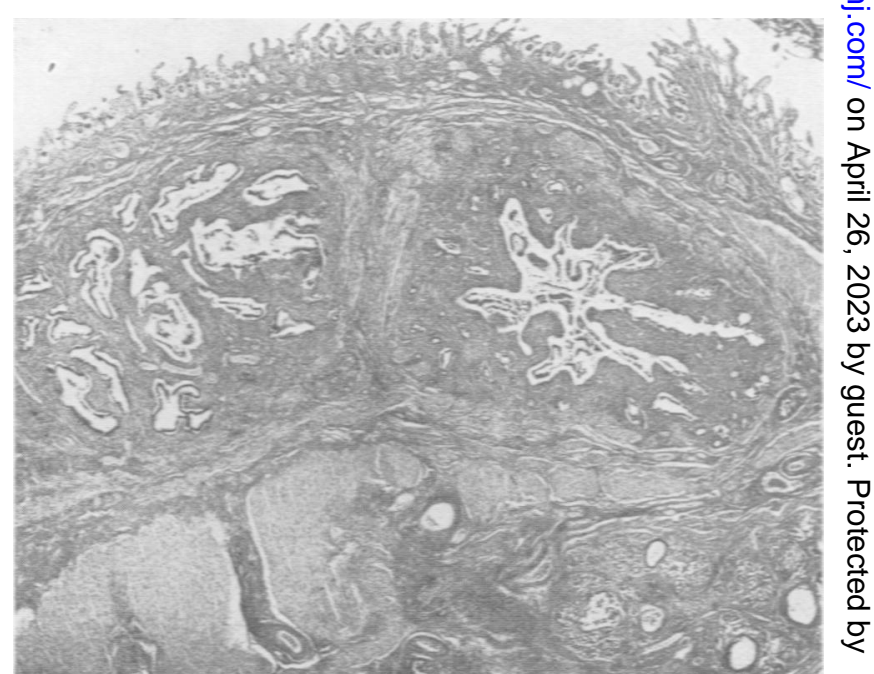

FIG. 4. 


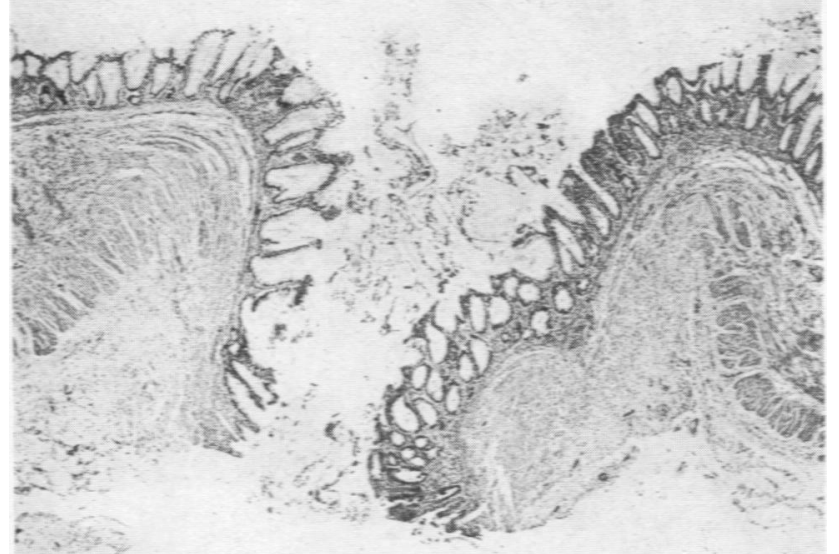

FIG. 5.
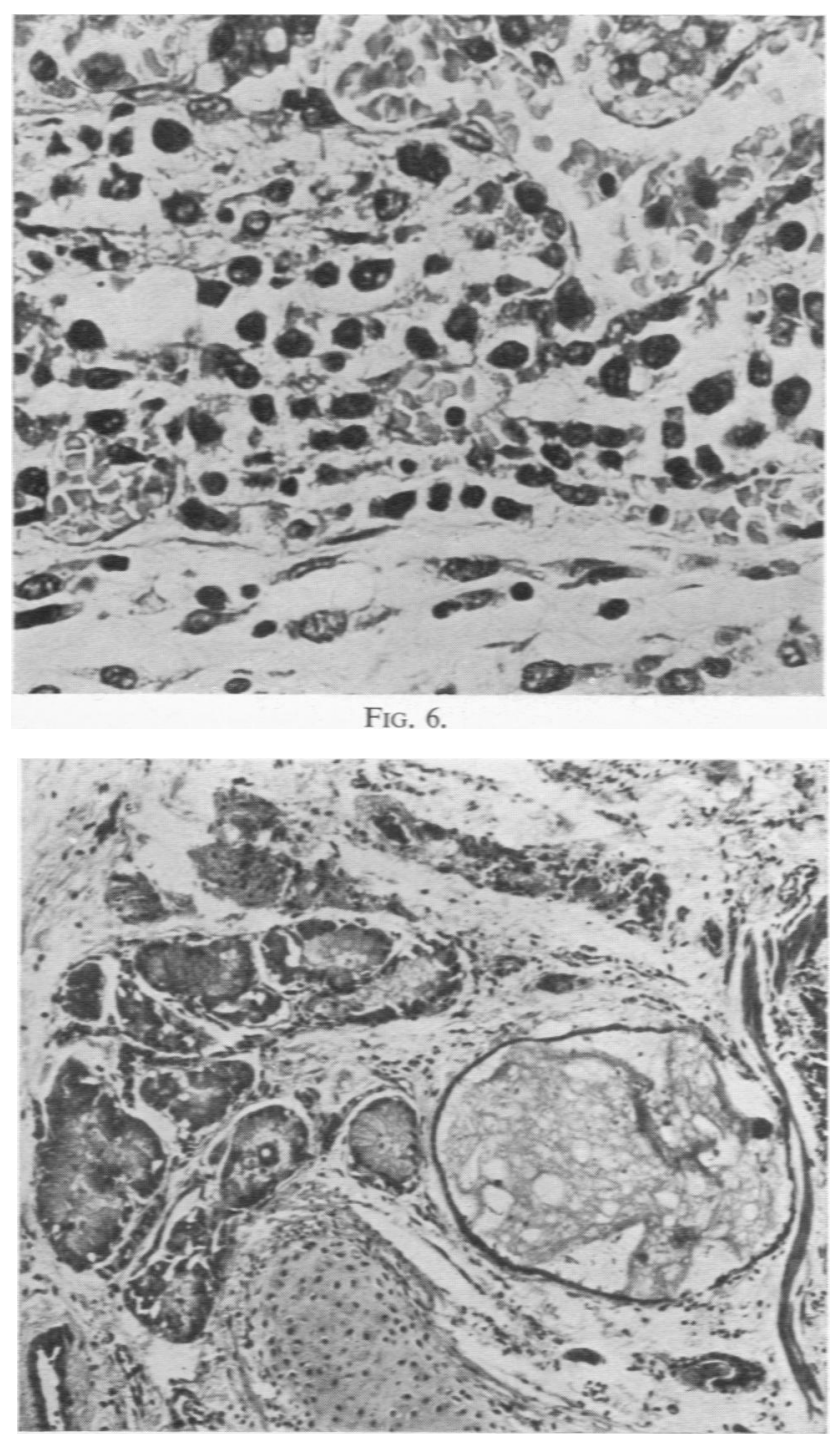

FIG. 7. stenosis or obstruction by bands, to a combination of mechanical factors and impaction and inspissation of normal meconium (Farber, 1944a), or to impaction and inspissation of abnormal meconium alone. In the present example the residual meconium in the intestine appeared abnormal to the naked eye and the change in the pancreas is important circumstantial evidence in favour of this. At necropsy definite evidence of intestinal obstruction was not found; but the perforation in the colon was of the 'blow through' type, such as can occur at a weak point in the intestinal wall above an obstruction. Such a weak point would be the place of penetration through the muscularis of entering vessels. The splenic flexure is an obvious place where abnormal meconium might become impacted, but this is an uncommon finding in cases of meconium ileus, in which the site of obstruction is usually at the lower end of the ileum.

We have been able to trace reports of 12 cases of intestinal obstruction associated with cystic fibrosis of the pancreas in the newborn, and of these, that of Kornblith and Otani (1929) is the only one in which impaction of meconium was thought to have occurred in the large intestine. In this case there was also evidence of obstruction at the terminal ileum, which was the site of obstruction in ten of the others; the twelfth, one of those reported by Andersen (1938), was an example of atresia of the jejunum. In the case of Kornblith and Otani there were two perforations of the 'blow through' type in the ascending colon and also a false diverticulum. These authors postulated an obstruction at the hepatic flexure but, as in the present instance, there was no definite evidence of this at necropsy. They discussed the mechanism of perforation in some detail, suggesting that false diverticula first form and then perforate. Active peristalsis is known to occur in the foetus after the fifth month of intrauterine life (Windle, 1940), hence prenatal obstruction and perforation of the intestine is possible. Abnormal meconium probably plays some part in the perforation but it is unlikely that it could penetrate the wall of the colon in the absence of obstruction.

Landsteiner (1905) was the first to report the association of meconium peritonitis with fibrocystic

Fig. 5.-Perforation in transverse colon. H. \& E. . 17.

Fig. 6.-Exudate on surface of liver. Numerous mononuclear cells between dilated capillaries. H. \& E. 480.

Fig. 7.-Main bronchus to lower lobe of left lung. Retained mucus in dilated duct of mucus gland. H. \& E. 97. 
disease of the pancreas. It is now generally accepted that the abnormal meconium is due to an absence of pancreatic secretion from the intestine, although recent evidence suggests that an abnormal protein in the meconium may be responsible for its peculiar consistency and the difficulty of its passage along the intestine (Glanzmann and Berger, 1950). There are two main theories concerning the mechanism of the retention of secretion within the pancreas. The well documented examples of Kornblith and Otani (1929) and of Hurwitt and Arnheim (1942) are usually accepted as cases of meconium ileus in which fibrocystic disease of the pancreas was due to a stenosis of the terminal part of the duct of Wirsung of developmental origin. In the present material, serial sections of this area showed a curious and striking splitting of the lumen of the duct into clefts separated by fibrous tissue septa (Fig. 4) but there still appeared to be ample space for the free passage of secretion. Reference to the paper of Kirk (1944) on the normal anatomy of the common opening of the pancreatic and bile ducts into the duodenum suggested that this appearance was normal. Examination by serial sections of a similar area in a control full term foetus showed an essentially similar appearance, although the number of separate lumina in the control was smaller, possibly because in the latter the sections were at right angles to the axis of the duct, whereas in the material from the case under investigation the sections were partly tangential. Kirk (1944), in his description of the mucosa in the common opening of the ducts into the duodenum, compares the arrangement to the plication of the oviduct and suggests that it may have some function in preventing duodenal fluid regurgitating into the ducts. We would agree with this and do not consider that this plication causes obstruction. The appearances resemble in some ways those described by Kornblith and Otani (1929), and Hurwitt and Arnheim (1942), but the striking dilatation of the duct in the body of the pancreas described by them was not seen. There is no evidence from the present material of a mechanical obstruction in the main pancreatic duct. Baggenstoss, Power and Grindlay (1951) have claimed that there is atresia of numerous interlobular ducts and that this accounts for the changes in the pancreas. In our material some of the smaller ducts appeared to end blindly in fibrous tissue, but there was no indication of any persistent solid columns of epithelial cells such as might be expected in atresia. We think it more likely that secondary obliteration of ducts occurs. The explanation of Farber (1944b) that the obstruction and consequent dilatation of smaller ducts is due to an abnormal inspissated secretion appears to us to be more likely. This author mentions the frequent occurrence of abnormal secretions in other glandular tissues. We can find no definite evidence of this in our case. Secretion within mucous glands of the trachea and the main bronchus to the left lower lobe is abundant, but not more excessive than has been seen in some controls of the same age dying with respiratory infection or after inhalation of foreign material. In this infant, asphyxia was present at birth and the sections of lung showed hyaline necrotic material in many small bronchi, so that inhalation of amniotic fluid may have occurred. Furthermore, a general anaesthetic with endotracheal intubation had been given. No other indication of retention of secretion was seen in the many tissues examined, except for distension of one gland in the appendix, and this might have been caused by the intestinal obstruction. Unfortunately examination of salivary glands was omitted.

The clinical features of our case were of some interest. On admission, examination of the child's abdomen showed no true guarding or rigidity. Moreover, his general condition remained good until the time when the abdomen was opened and was found to be full of purulent fluid. These features are in keeping with one's experience of peritonitis in small infants, that is to say the clinical signs of peritonitis may be lacking and the general picture unrelated to the degree of peritoneal inflammation found at operation or necropsy. They may, perhaps, be explained by the fact that, although meconium peritonitis was probably present at birth, bacterial infection would not have taken place until after delivery. In addition, the fluid requirements of such a young infant would be small, and in this particular child fluid loss, apart from the formation of peritoneal exudate, was negligible. The absence of vomiting was noteworthy, and suggests that perforation of the colon and relief of intestinal obstruction had occurred at or before delivery; the appearance of the peritoneal exudate at necropsy lends support to this view.

\section{Summary}

A case of meconium peritonitis in association with fibrocystic disease of the pancreas in a newborn infant is reported.

Careful examination of the main pancreatic duct by serial section revealed no mechanical obstruction or stenosis.

Features of particular interest were the presence of abdominal distension at birth; the absence of vomiting, which is usually a prominent feature of most cases of meconium ileus; and the unusual site of intestinal perforation. 
Reasons are given for our view that this perforation nccurred before or during labour.

We are indebted to Dr. R. H. Dobbs for permission to publish this case and for helpful criticism, to Professor D. S. Russell for advice, and to Professor J. D. Boyd for information concerning the normal development and anatomy of the pancreatic ducts. We are also grateful to the technicians of the Department of Anatomy who prepared the serial sections.

\section{REFERENCES}

Abt, I. A. (1931). Med. Clin. N. Amer., 15, 611. Andersen, D. H. (1938). Amer. J. Dis. Child., 56, 344.

Baggenstoss, A. H., Power, M. H. and Grindlay. J. H. (1951). Arch. Path., Chicago, 51, 510.

Farber, S. (1944a). J. Pediat., 24, 387. (1944b). Arch. Path., Chicago, 37, 238.

Glanzmann, E. and Berger, H. (1950). Ann. paediatr., Basel, 175, 33.

Hurwitt, E. S., and Amheim, E. E. (1942). Amer. J. Dis. Child., 64, 443.

Kirk, J. (1944). J. Anat., Lond., 78, 118.

Kornblith, B. A. and Otani, S. (1929). Amer. J. Path., $5,249$.

Landsteiner, K. (1905). Zbl. allg. Path. path. Anat., 16, 903.

Simpson, J. Y. (1838). Edinb. med. surg. J., 50, 390.

Thelander, H. E. (1939). Amer. J. Dis. Child., 58, 371.

Windle, W. F. (1940). 'Physiology of the Fetus, Ch. 7. Philadelphia. 